\title{
Sites of active gene regulation in the prenatal frontal cortex and their role in neuropsychiatric disorders
}

\author{
Manuela R. Kouakou ${ }^{1}$ | Darren Cameron ${ }^{1}$ | Eilis Hannon ${ }^{2}$ | Emma L. Dempster ${ }^{2}$ | \\ Jonathan Mill $^{2}$ | Matthew J. Hill ${ }^{1}$ | Nicholas J. Bray ${ }^{1}{ }^{\infty}$
}

${ }^{1}$ MRC Centre for Neuropsychiatric Genetics and Genomics, Division of Psychological Medicine and Clinical Neurosciences, Cardiff University, Cardiff, UK

${ }^{2}$ University of Exeter Medical School, University of Exeter, Exeter, UK

\section{Correspondence}

Nicholas J. Bray, MRC Centre for

Neuropsychiatric Genetics and Genomics, Cardiff University School of Medicine, Hadyn Ellis Building, Maindy Road, Cardiff CF24 4HQ, UK.

Email: brayn3@cardiff.ac.uk

\section{Funding information}

Joint MRC/Wellcome Trust, Grant/Award Number: \#099175/Z/12/Z; MRC Centre, Grant/Award Number: MR/L010305/1; MRC project, Grant/Award Number: MR/ L010674/2

\begin{abstract}
Common genetic variation appears to largely influence risk for neuropsychiatric disorders through effects on gene regulation. It is therefore possible to shed light on the biology of these conditions by testing for enrichment of associated genetic variation within regulatory genomic regions operating in specific tissues or cell types. Here, we have used the assay for transposase-accessible chromatin with high-throughput sequencing (ATAC-Seq) to map open chromatin (an index of active regulatory genomic regions) in bulk tissue, $\mathrm{NeuN}+$ and $\mathrm{NeuN}$ - nuclei from the prenatal human frontal cortex, and tested enrichment of single-nucleotide polymorphism (SNP) heritability for five neuropsychiatric disorders (autism spectrum disorder, attention deficit hyperactivity disorder [ADHD], bipolar disorder, major depressive disorder, and schizophrenia) within these regions. We observed significant enrichment of SNP heritability for ADHD, major depressive disorder, and schizophrenia within open chromatin regions (OCRs) mapped in bulk fetal frontal cortex, and for all five tested neuropsychiatric conditions when we restricted these sites to those overlapping histone modifications indicative of enhancers ( $\mathrm{H} 3 \mathrm{~K} 4 \mathrm{me} 1)$ or promoters $(\mathrm{H} 3 \mathrm{~K} 4 \mathrm{me} 3)$ in fetal brain. SNP heritability for neuropsychiatric disorders was significantly enriched in OCRs identified in fetal frontal cortex $\mathrm{NeuN}$ - as well as NeuN+ nuclei overlapping fetal brain H3K4me1 or H3K4me3 sites. We additionally demonstrate the utility of our mapped OCRs for prioritizing potentially functional SNPs at genome-wide significant risk loci for neuropsychiatric disorders. Our data provide evidence for an early neurodevelopmental component to a range of neuropsychiatric conditions and highlight an important role for regulatory genomic regions active within both $\mathrm{NeuN}+$ and $\mathrm{NeuN}$ - cells of the prenatal brain.
\end{abstract}

\section{KEYWORDS}

gene expression, gene regulation, GWAS, psychiatric genetics, schizophrenia

\section{1 | INTRODUCTION}

The majority of common genetic variants implicated in neuropsychiatric disorders are located in regions of the genome that do not encode proteins (Demontis et al., 2019; Grove et al., 2019; Mullins et al., 2021; Ripke, Walters, \& O'Donovan, 2020; Wray et al., 2018). These noncoding genomic regions contain cis-regulatory elements such as gene promoters, enhancers, and silencers that are variably 
utilized in order to control gene expression in a cell-specific manner (ENCODE Project Consortium, 2012; Roadmap Epigenomics Consortium, 2015). It is possible to map regulatory genomic sites operating in a given tissue or cell type based on epigenomic features. These include "open," or "accessible," chromatin, which exposes DNA to the transcription factors that modulate gene expression (Tsompana \& Buck, 2014). As regulatory regions are believed to contain much of the common genetic component of complex traits, tissues and cell types that are relevant to the etiology of a trait can be delineated by testing for enrichment of trait-associated genetic variation within cell-specific epigenomic features (Finucane et al., 2015).

Several neuropsychiatric disorders are hypothesized to have origins in early brain development (Courchesne, Gazestani, \& Lewis, 2020; O'Donnell \& Meaney, 2017; Weinberger, 1987). Consistent with this view, we have recently found that genetic variants associated with altered gene expression (expression quantitative trait loci [eQTL]) in the human fetal brain are enriched among risk variants for ADHD, bipolar disorder, and schizophrenia (O'Brien et al., 2018). Similarly, de la Torre-Ubieta et al. (2018) mapped genomic regions of open chromatin in the germinal zone and cortical plate of the fetal cerebral cortex, reporting significant enrichment of genetic variation associated with ADHD, depressive symptoms, neuroticism, and schizophrenia within sites defined as preferentially accessible in the germinal zone. In the present study, we used the assay for transposaseaccessible chromatin with high-throughput sequencing (ATAC-Seq; Buenrostro, Giresi, Zaba, Chang, \& Greenleaf, 2013) to map regions of open chromatin in the human second-trimester frontal cortex, finding these to be enriched for genetic variation associated with a range of neuropsychiatric disorders, particularly when histone modifications indicative of enhancers or promoters were also considered. In addition, we provide the first maps of open chromatin in $\mathrm{NeuN}+$ (neuronenriched) and $\mathrm{NeuN}$ - (neuron-depleted) nuclei from the human fetal brain, and demonstrate the use of our open chromatin maps for prioritizing potentially functional single-nucleotide polymorphisms (SNPs) at genome-wide significant risk loci for neuropsychiatric disorders.

\section{2 | METHODS}

\section{1 | Samples}

Open chromatin annotations were derived from three fresh human fetal frontal cortex samples from the second trimester of gestation (16, 18, and 19 postconception weeks). This number of biological replicates is consistent with other epigenomic studies of its kind (e.g., de la Torre-Ubieta et al., 2018) and meets current ENCODE ATAC-Seq standards for genomic annotation, which stipulate at least two independent replicates. Fetal cortex samples were acquired from the MRC-Wellcome Trust Human Developmental Biology Resource (HDBR) (http://www.hdbr.org/) in Hibernate-E media (Thermo Fisher Scientific). All samples were obtained through elective terminations of pregnancy, with consent from female donors, and were of normal karyotype (two females and one male). Ethical approval for the collection and distribution of fetal material for scientific research was granted to the HDBR by the Royal Free Hospital research ethics committee (reference 08/H0712/34) and NRES Committee North East-Newcastle and North Tyneside (reference 08/H0906/21+5). The left frontal cortex from each fetus was dissected and dounce homogenized on ice to produce a single-cell suspension. Prior to nuclei isolation, aliquots of 1-10 million cells were stored at $-80^{\circ} \mathrm{C}$ in cryovials containing $1 \mathrm{ml}$ Hibernate-E media, supplemented with $6 \%$ dimethyl sulfoxide, in Nalgene ${ }^{\circledR}$ Mr Frosty containers.

\section{2 | Nuclei isolation and assay for transposase- accessible chromatin in bulk tissue}

Two technical replicates were processed from each of the three bulk frontal cortex samples. Nuclei were isolated from cryopreserved cell suspensions by first centrifuging cells at $600 \mathrm{~g}$ for $5 \mathrm{~min}$ and resuspending them in ice-cold cell lysis buffer (sucrose $0.25 \mathrm{M} ; \mathrm{KCl}$ $25 \mathrm{mM}, \mathrm{MgCl}_{2} 5 \mathrm{mM}$, Tris-Cl $10 \mathrm{mM}$, dithiothereitol $1 \mathrm{mM}, 0.1 \%$ Triton) for $15 \mathrm{~min}$. Nuclei were then pelleted by centrifuging at $1000 \mathrm{~g}$ for $8 \mathrm{~min}$ and resuspended in storage buffer (sucrose $0.25 \mathrm{M}, \mathrm{MgCl}_{2}$ $5 \mathrm{mM}$, Tris-Cl $10 \mathrm{mM}$, bovine serum albumin [BSA] $0.1 \%$ ) by gentle pipetting for $10 \mathrm{~s}$. The nuclei suspension was then left on ice for $10 \mathrm{~min}$ and passed through a $21 \mathrm{G}$ needle 10 times to thoroughly resuspend nuclei. Nuclei were then visualized under an optical microscope for quality control and counting. For each technical replicate, 50,000 nuclei were incubated in the transposition reaction mix $(20 \mu \mathrm{l}$ nuclease-free water; $25 \mu \mathrm{l} 2 \mathrm{X}$ Tagment DNA Buffer, Illumina Cat\#FC121-1030; $5 \mu \mathrm{l}$ Tn5 Transposase, Illumina Cat\#FC-121-1030) at $37^{\circ} \mathrm{C}$ for $30 \mathrm{~min}$, as described by Buenrostro et al. (2013).

\section{3 | Fluorescence-activated nuclei sorting (FANS)}

Nuclei were isolated from cryopreserved frontal cortex cell suspensions as described above. To block nonspecific antibody binding, the resulting nuclei pellet was resuspended in buffer containing sucrose $0.25 \mathrm{M}, \mathrm{MgCl}_{2} 5 \mathrm{mM}$, Tris-Cl $10 \mathrm{mM}$, and BSA 1\% and incubated for $30 \mathrm{~min}$ on ice. Nuclei were visualized under an optical microscope for quality control and counting. The samples were then centrifuged at $400 \mathrm{~g}$ for $8 \mathrm{~min}$ and the nuclei pellet resuspended in FANS buffer (0.5\% BSA in Dulbecco's phosphate-buffered saline).

Before immunostaining, $100 \mu$ of the sample was transferred to a new tube and used as unstained control in the FANS analysis. The remaining sample was incubated with mouse anti-neuronal nuclei (NeuN) Alexa Fluor 488-conjugated monoclonal antibody (Merck Millipore; Cat\# MAB377X), at a 1:2,000 dilution, and rotated at $4^{\circ} \mathrm{C}$ in the dark for $60 \mathrm{~min}$. After immunostaining, samples were washed three times in FANS buffer $(400 \mathrm{~g}$ for $5 \mathrm{~min}$ ) to remove excess antibody. 4',6-Diamidino-2-phenylindole (DAPI) was then added to a final concentration of $1 \mu \mathrm{g} / \mathrm{ml}$ and the nuclei suspension was filtered through a $35-\mu \mathrm{m}$ cell strainer to remove nuclei clumps and prevent clogging of the cytometer. DAPI-positive neuronal $(\mathrm{NeuN}+)$ and 
nonneuronal (NeuN-) nuclei were sorted into tubes precoated with 5\% BSA using a FACSAria flow cytometer (BD Biosciences) equipped with a $100-\mu \mathrm{m}$ nozzle. For both flow analysis and FANS, exclusion of debris using forward and side scatter pulse area parameters (FSC-A and SSC-A) were gated first, followed by exclusion of aggregates using pulse width (FSC-W and SSC-A) and DAPI-positive nuclei before gating populations based on NeuN fluorescence (Figure S1). Sorted $\mathrm{NeuN}+$ and $\mathrm{NeuN}$ - nuclei were incubated in transposition reaction mix and polymerase chain reaction (PCR) amplified as for bulk tissue. Sufficient nuclei were recovered to perform two technical replicates for two of the three frontal cortex samples and one technical replicate for the other, resulting in five separate transposase reactions for $\mathrm{NeuN}+$ nuclei (mean number of nuclei per reaction $=41,216$ ) and five separate transposase reactions for $\mathrm{NeuN}$ - nuclei (mean number of nuclei per reaction $=44,066$ ).

\section{4 | PCR amplification, size selection, and sequencing of ATAC-Seq libraries}

Transposase reactions were initially amplified by eight cycles of PCR using primers described by Buenrostro et al. (2013) and 2X NEBNext Q5 HotStart HiFi Master Mix (New England Biolabs; Cat\# M0543). Amplicons of $175-250$ bp were size-selected using agarose gel electrophoresis on BluePippin 2\% gel cassettes (Sage Science) and further amplified through seven additional PCR cycles. Fragment size analysis and quantification of ATAC-seq libraries were performed using an Agilent Technologies 2100 Bioanalyzer and high sensitivity DNA kit and the Qubit dsDNA HS Assay Kit (Thermo Fisher Scientific; Cat\#32854). ATAC-Seq libraries were sequenced on an Illumina HiSeq 4000 to a depth of at least 100 million paired-end 75 bp reads per library.

\section{5 | ATAC-Seq data analysis}

Sequencing quality was confirmed using FastQC (http://www. bioinformatics.babraham.ac.uk/projects/fastqc). Reads were aligned to the GRCh37 (hg19) human genome reference sequence using Bowtie2 v2.2.9 (Langmead, Trapnell, Pop, \& Salzberg, 2009) following adapter trimming. This produced a SAM file for each replicate, which was then converted into a coordinate-sorted BAM file of paired-end reads with Samtools v1.5 (Li et al., 2009). Reads that mapped to more than one locus or were PCR duplicates were excluded. This yielded >90 million uniquely mapped, nonduplicated paired-end reads per technical replicate.

We first downsampled reads from the six BAM files (two technical replicates for each of the three biological replicates) for bulk fetal frontal cortex to the lowest read count of any technical replicate and merged these using Samtools v1.5 (Li et al., 2009) to produce a large, single downsampled BAM file representing all samples. Open chromatin peaks (FDR < 0.01) were identified in the downsampled bulk tissue BAM file using MACS2 (Zhang et al., 2008), while artifact regions (defined as "Blacklist" regions by ENCODE; https://www. encodeproject.org/files/ENCFF001TDO/) with excessive unstructured anomalous read mapping (e.g., regions of centromeres, telomeres, and satellite repeats) were excluded. We then merged the two technical replicate BAM files for each biological replicate to obtain three biological replicate BAM files. MACS2 (Zhang et al., 2008) was used to identify peaks (FDR $<0.01$ ) in each biological replicate and DiffBind v2.16.0 (Stark \& Brown, 2011) to determine peaks observed in at least two of the three biological replicates. To generate a set of high confidence bulk fetal frontal cortex open chromatin regions (OCRs) for our main analyses, we selected peaks (FDR $<0.01$ ) in the single downsampled BAM file that intersected with the (FDR < 0.01$)$ peaks observed in at least two of the three biological replicates. The five technical replicate BAM files for $\mathrm{NeuN}+$ and $\mathrm{NeuN}$ - nuclei were similarly downsampled and merged before identifying open chromatin peaks (FDR < 0.01) using MACS2 (Zhang et al., 2008). To generate subsets of bulk fetal frontal cortex OCRs that could be attributed to the $\mathrm{NeuN}+$ and/or NeuN - fractions, we selected those high confidence bulk peaks that intersected with the FDR $<0.01$ peaks identified in the merged BAM files for both $\mathrm{NeuN}+$ and $\mathrm{NeuN}$ - nuclei.

\subsection{Overlap between OCRs identified in different tissues}

ChIPpeakAnno (Zhu et al., 2010) was used to determine the numbers of bulk fetal frontal OCRs overlapping adult frontal cortex OCRs (Hoffman et al., 2019) and OCRs identified in the germinal zone/ cortical plate of the fetal cortex (de la Torre-Ubieta et al., 2018). Consensus OCRs identified by ATAC-Seq in bulk adult frontal cortex (CMC_ATACSeq_consensusPeaks.bed) were downloaded from https://www.synapse.org/ and converted to GRCh37 coordinates using https://genome.ucsc.edu/cgi-bin/hgLiftOver. OCRs identified by ATAC-Seq in the germinal zone/cortical plate of the fetal cortex were downloaded from https://www.ncbi.nlm.nih.gov/geo/query/ acc.cgi?acc=GSE95023.

\subsection{Bioinformatic annotation of OCRs}

We tested for overlap between bulk fetal frontal cortex OCRs and chromatin states that have been defined in independent samples of (bulk) human fetal brain and regions of the adult human brain on the basis of other epigenomic assays (histone modification ChIP-Seq, transcription factor ChIP-Seq and DNAse-Seq; Libbrecht et al., 2019). Annotation maps were downloaded from https://noble.gs. washington.edu/proj/encyclopedia/interpreted/ and the R package ChIPseeker (Yu, Wang, \& He, 2015) used to simulate 5,000 sets of regions that matched the genomic distribution of the bulk fetal frontal cortex OCRs in terms of chromosome distribution and region sizes. Taking all regions annotated to the same chromatin state for each sample in turn, we counted the number of overlapping peaks with the simulated set of OCR peaks. An overlap was defined as $>50 \%$ of the 
OCR peak intersecting an annotated region. The mean number of intersecting regions calculated across all simulations was compared to the true overlap to calculate a fold-enrichment statistic. One-sided empirical $p$-values for both over- and under-enrichment were calculated separately as the number of simulations with a more extreme overlap divided by the total number of simulations, adding 1 to both the denominator and numerator. Two-sided $p$-values were calculated as (a) the sum of the one-sided $p$-value for over-enrichment and 1 minus the one-sided $p$-value for under-enrichment, if the foldchange was $>1$, or (b) the sum of one-sided $p$-value for underenrichment and 1 minus the one-sided $p$-value for over-enrichment, if the fold-change was $<1$.

We tested for enrichment of transcription factor binding motifs within high confidence fetal frontal cortex OCRs using HOMER (http://homer.ucsd.edu/homer/ngs/peakMotifs.html). Enrichment of biological process Gene Ontology (GO) annotations for genes with fetal frontal cortex OCRs within $30 \mathrm{~kb}$ upstream and $100 \mathrm{bp}$ downstream of their transcription start site (TSS) was tested using g:Profiler (Raudvere et al., 2019), correcting for multiple testing using the default g:SCS algorithm. We tested for enrichment of previously identified genome-wide significant $\left(p<5 \times 10^{-8}\right)$ eQTL (O'Brien et al., 2018) and methylation quantitative trait loci (mQTL) (Hannon et al., 2016) operating in the human fetal brain within fetal frontal cortex OCRs using GARFIELD (Iotchkova et al., 2019), a method that controls for minor allele frequency, GC-content, linkage disequilibrium, and local gene density.

\section{8 | Histone modification datasets from human fetal brain}

Genomic regions marked by $\mathrm{H} 3 \mathrm{~K} 4 \mathrm{me} 1$ or $\mathrm{H} 3 \mathrm{~K} 4 \mathrm{me} 3$ in a bulk human fetal brain sample of similar gestational age to those used in this study (sample E082; female, 17 postconception weeks) were downloaded as BED files from the Roadmap Epigenomics Project (https://egg2.wustl. edu/roadmap/data/byFileType/peaks/consolidated/narrowPeak/). BEDTools v2.26.0 intersect and merge (Quinlan \& Hall, 2010) were used to identify and merge $\mathrm{H} 3 \mathrm{~K} 4 \mathrm{me} 1$ and $\mathrm{H} 3 \mathrm{~K} 4 \mathrm{me} 3$ sites overlapping the OCRs that we identified in nuclei from the fetal frontal cortex.

\section{9 | Testing enrichment of SNP heritability for neuropsychiatric disorders in OCRs}

Stratified linkage disequilibrium score regression (SLDSR; Finucane et al., 2015) was used to test for enrichment of SNP heritability for neuropsychiatric disorders within fetal brain OCRs, H3K4me1 sites, H3K4me3 sites, and OCRs overlapping these sites. Peaks were expanded by $500 \mathrm{bp}$ on each side prior to SLDSR, as recommended by Finucane et al. (2015). Summary statistics from large-scale genome-wide association study (GWAS) of five neuropsychiatric disorders (ADHD, Demontis et al., 2019; autism spectrum disorder [ASD], Grove et al., 2019; bipolar disorder, Mullins et al., 2021; major depressive disorder, Wray et al., 2018; schizophrenia, Ripke et al., 2020) and two control traits with similar sample sizes (height, Lango Allen et al., 2010; blood triglyceride levels, Teslovich et al., 2010) were tested (Table S6). Fold-enrichment estimates are the proportion of SNP heritability for each trait explained by SNPS within the annotation divided by the proportion of genome-wide SNPs within the annotation. Each annotation was also assessed against the baseline model provided by Finucane et al (consisting of 53 genomic annotations including coding regions, promoters, enhancers, and conserved regions) and resulting Z-scores used to calculate two-tailed $p$-values. We more conservatively state the Z-score $p$-values, rather than raw fold-enrichment $p$-values, in the text and figures, highlighting those that survive Bonferroni correction for the seven tested traits (i.e., $p<.0071$ ).

\subsection{0 | Identification of SNPs within OCRs in strong linkage disequilibrium with index SNPs from GWAS of neuropsychiatric disorders}

We used GARFIELD (lotchova et al., 2019) to identify SNPs within high confidence bulk fetal frontal cortex OCRs that exhibit genomewide significant $\left(p<5 \times 10^{-8}\right)$ association with any of the five tested neuropsychiatric disorders in the associated GWAS. We used LDpair within the NIH national Cancer Institute LDlink (https://Idlink.nci.nih. gov/) to further identify those OCR SNPs that were in strong linkage disequilibrium $\left(r^{2}>.8\right)$ with the most significant (index) SNP at each genome-wide significant risk locus in the CEU population. To further prioritize potentially functional OCR SNPs, we identified those for which there was evidence that they act as eQTL in the human fetal brain ( $p<5 \times 10^{-5}$; O'Brien et al., 2018), and used LDpair to determine the $r^{2}$ between them and the most significant eQTL for the implicated transcript in the CEU population.

\section{3 | RESULTS}

We used ATAC-Seq (Buenrostro et al., 2013) to map OCRs in the frontal cortex of three human fetuses from the second trimester of gestation. We first profiled OCRs within unsorted nuclei from these samples ("bulk" frontal cortex analyses), identifying 88,501 high confidence OCRs using stringent peak calling criteria (see Section 2), and observing strong correlations in the number of reads within peaks between biological replicates (median $r=.83 ; p<2.2 \times 10^{-16}$; Figure S2). The genomic distribution of peaks approximated that of OCRs identified in other tissues (Bryois et al., 2018; Song et al., 2011), with $28 \%$ within $5 \mathrm{~kb}$ of a TSS and $38 \%$ within gene bodies. Exemplar fetal frontal cortex OCRs, located at the TSS and first intron of the gene encoding the neurogenic transcription factor NeuroD1, are shown in Figure 1a. Identified OCRs were prominently enriched in genomic regions annotated as active promoters and enhancers (Libbrecht et al., 2019) on the basis of histone modifications and other epigenomic features in independent bulk human fetal brain samples 
(a)
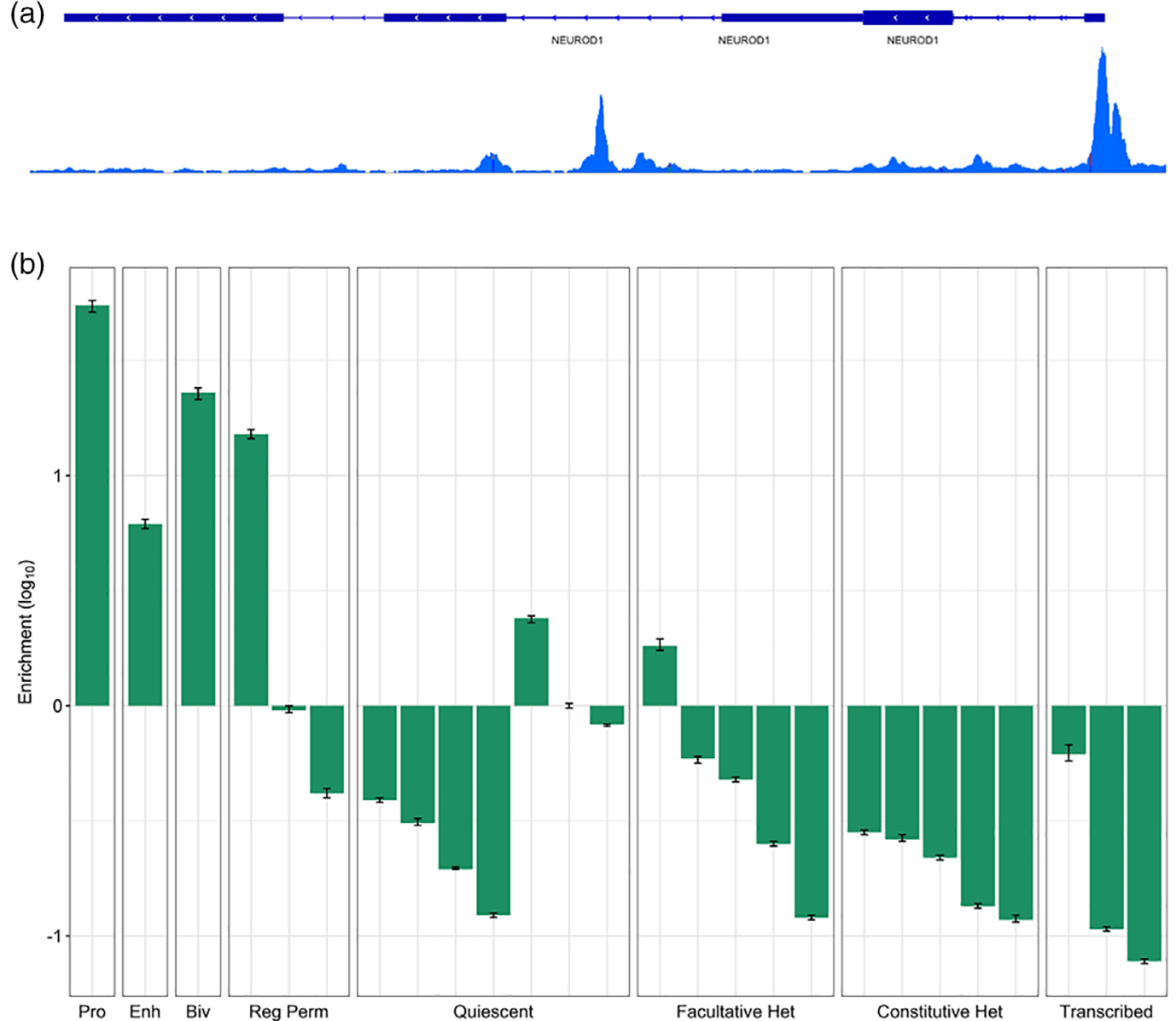

(c)

(d)
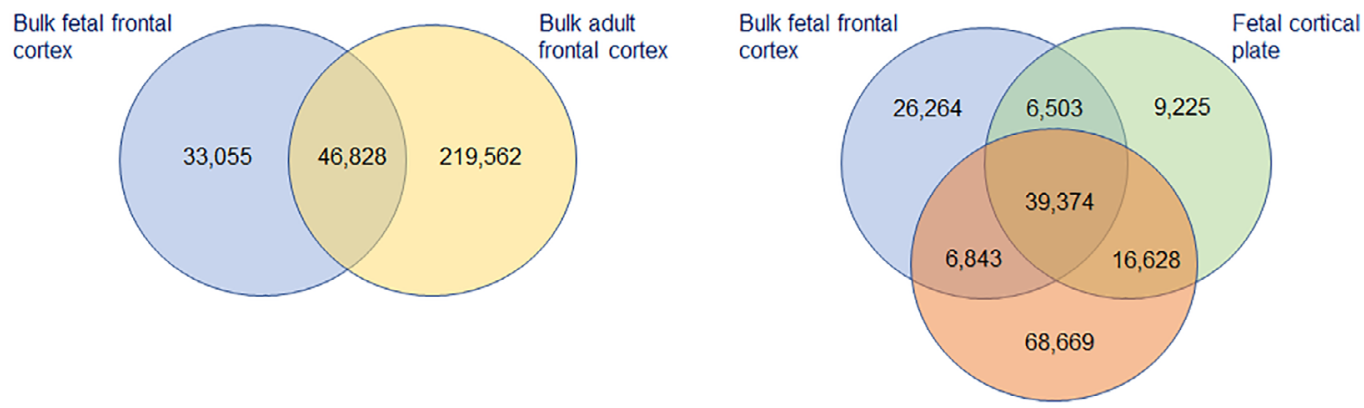

Fetal germinal zone

FIGURE 1 Characteristics of high confidence bulk fetal frontal cortex open chromatin regions. (a) Combined reads from the three bulk fetal frontal cortex samples showing a high confidence open chromatin region at the transcription start site of the NEUROD1 gene. (b) Log 10 enrichments of fetal frontal cortex open chromatin regions within functional genomic annotations defined by Libbrecht et al. (2019) in bulk human fetal brain based on histone modifications and other epigenomic features. Pro, promoter; Enh, enhancer; Biv, bivalent: regulatory element with marks of both activation (such as H3K27ac) and repression (H3K27me3); Reg Perm, RegPermissive: region with weak marks of regulatory activity such as H3K4me1 or DNase hypersensitivity; Quiescent: inactive region; Facultative Het: heterochromatin marking regions of cell typespecific repression, characterized by the histone modification H3K27me3 (also known as polycomb-repressed heterochromatin); Constitutive Het: heterochromatin marking permanently silent regions, characterized by the histone modification H3K9me3; Transcribed: transcribed genic region. (c) Overlap between open chromatin regions identified in bulk fetal frontal cortex and bulk adult frontal cortex (Hoffman et al., 2019). (d) Overlap between open chromatin regions identified in bulk fetal frontal cortex and those identified in cortical plate and germinal zone of the fetal cortex in the study of de la Torre-Ubieta et al. (2018). Note that the minimum number of overlapping peaks is calculated, and therefore the summed number of open chromatin regions will usually be smaller than the total number in each set 
(54.3- and 6.1-fold enrichment respectively; $p<.0002$ ), while showing a general depletion in regions annotated as quiescent or repressed in the fetal and adult brain (Figure 1b; Table S1). Fetal frontal cortex OCRs were highly enriched for the CTCF binding motif (11.8-fold enrichment, $p=1 \times 10^{-5440}$ ), with notable enrichment of motifs for neural transcription factors such as Olig2 (2.2-fold enrichment, $p=1$ $\left.\times 10^{-1,067}\right)$ and Oct6 (2.1-fold enrichment, $\left.p=1 \times 10^{-449}\right)$ also observed. Consistent with roles in gene regulation, fetal frontal cortex OCRs were enriched for both eQTL (2.1-fold enrichment, $p=3.47$ $\left.\times 10^{-19}\right)$ and mQTL (2.3-fold enrichment, $p=1.88 \times 10^{-23}$ ) identified in the human fetal brain (Hannon et al., 2016; O'Brien et al., 2018).

Approximately $60 \%$ of the OCRs we identified in fetal frontal cortex overlapped OCRs observed in adult human frontal cortex (Hoffman et al., 2019; Figure 1c); genes with nonoverlapping "fetalspecific" OCRs within $30 \mathrm{~kb}$ upstream of their TSS were most significantly enriched for the GO term "nervous system development" ( $p$ corrected $=6.7 \times 10^{-9}$ ), while the larger number of genes with "adult-specific" OCRs within $30 \mathrm{~kb}$ upstream of their TSS were highly enriched for the GO terms "signaling" ( $p$ corrected $=2.6 \times 10^{-60}$ ) and "cell communication" ( $p$ corrected $=1.2 \times 10^{-59}$ ). There was also substantial overlap with OCRs previously observed in both the germinal zone and cortical plate of the human fetal brain (de la Torre-Ubieta et al., 2018; Figure 1d), although we note that $\sim 33 \%$ of our highconfidence fetal cortex OCRs were not identified in either of these regions.

We tested for enrichment of SNP heritability for five major neuropsychiatric disorders (ADHD, ASD, bipolar disorder, major depressive disorder, and schizophrenia) within bulk fetal frontal cortex OCRs using SLDSR (Finucane et al., 2015), controlling for general genomic annotations (e.g., coding regions, promoters, enhancers, and conserved regions) included in the baseline model to obtain Z-score $p$ values. We observed significant enrichment of SNP heritability for ADHD (5.6-fold enrichment, Z-score $p=.009$ ), major depressive disorder (4.4-fold enrichment, Z-score $p=.024$ ), and schizophrenia (7.2-fold enrichment, $Z$-score $p=1.2 \times 10^{-11}$ ) within these regions, the latter surviving Bonferroni correction for the seven tested traits (Table S2). In contrast, we observed no such enrichment of SNP heritability for either of the control traits (triglyceride levels or height) when baseline annotations were accounted for (Z-score $p>.1$ for both). The level of enrichment of schizophrenia risk variation in fetal frontal cortex OCRs approximates that reported in adult frontal cortex OCRs (Bryois et al., 2018), with 3.3\% of SNPs in fetal brain OCRs accounting for $23.9 \%$ of SNP heritability for the condition.
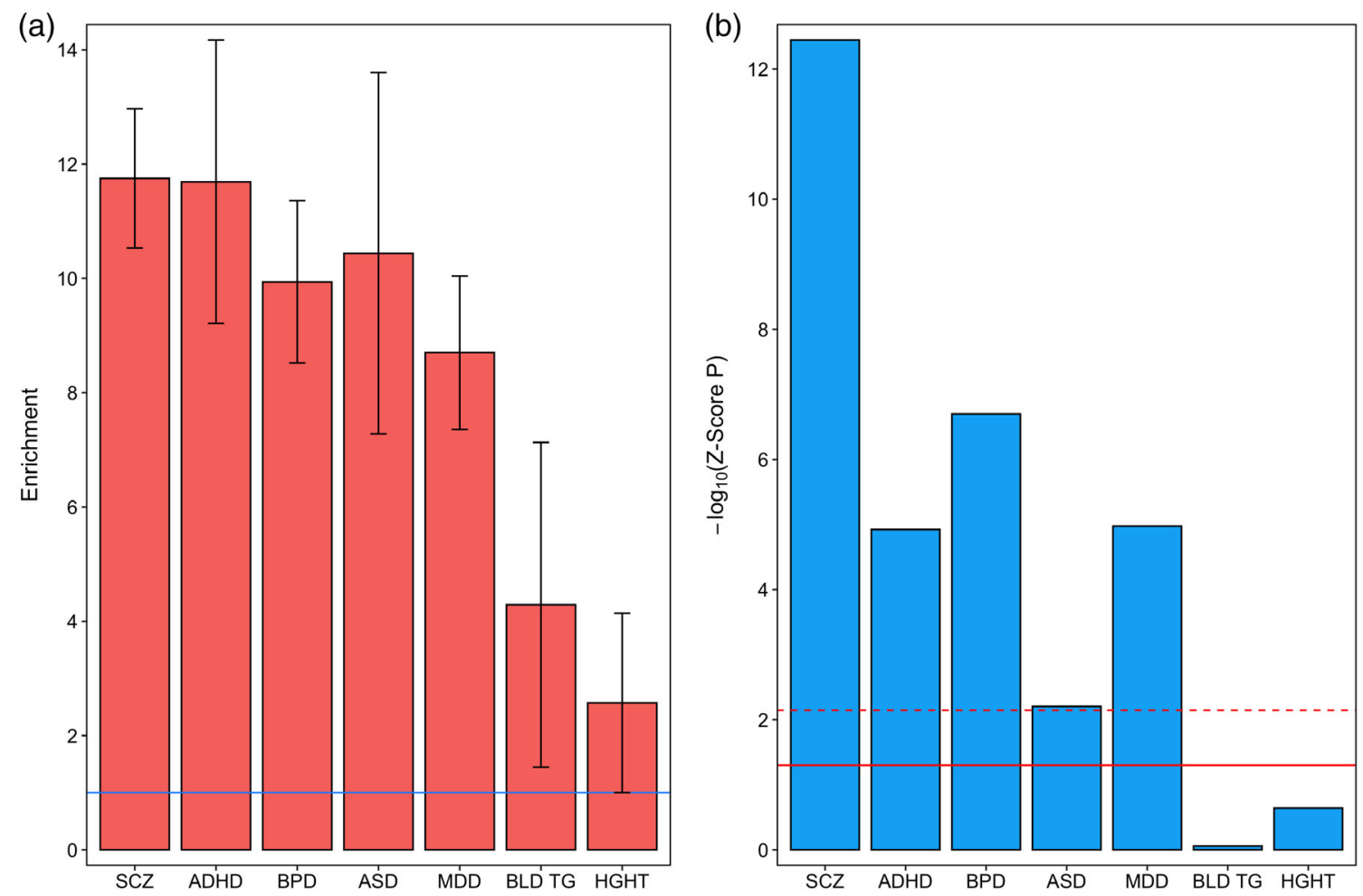

FIGURE 2 Partitioned heritability for five neuropsychiatric disorders and two control traits within open chromatin regions identified in bulk fetal frontal cortex overlapping fetal brain H3K4me1 sites. (a) Fold-enrichment estimates of SNP heritability for each trait (the proportion of SNP heritability explained by SNPs within the annotation divided by the proportion of genome-wide SNPs within the annotation). Error bars represent standard error. The solid horizontal line indicates no enrichment. (b) - $\log _{10} Z$-score $p$-values (two-tailed) for enrichment of SNP heritability, controlling for general genomic annotations included in the baseline model of Finucane et al. (2015). The solid horizontal line indicates the Zscore $p$-value .05 threshold; the dashed horizontal line indicates the threshold for Z-score $p$-values surviving Bonferroni correction for seven tested traits. ADHD, attention deficit hyperactivity disorder; ASD, autism spectrum disorder; BLD TG, blood triglyceride levels; BPD, bipolar disorder; HGHT, height; MDD, major depressive disorder; SCZ, schizophrenia 
A recent study (Schork et al., 2019) reported enrichment of SNP heritability for a broad neuropsychiatric phenotype encompassing ADHD, affective disorder, anorexia, ASD, bipolar disorder, and schizophrenia within $\mathrm{H} 3 \mathrm{~K} 4 \mathrm{me} 1$ and $\mathrm{H} 3 \mathrm{~K} 4 \mathrm{me} 3$ sites (histone modifications indicative of poised or active enhancers and promoters, respectively) identified by the Roadmap Epigenomics Mapping Consortium (2015) in bulk human fetal brain tissue. Consistent with these findings, when we restricted our bulk fetal frontal cortex OCRs to those overlapping either fetal brain $\mathrm{H} 3 \mathrm{~K} 4 \mathrm{me} 1$ or $\mathrm{H} 3 \mathrm{~K} 4 \mathrm{me} 3$ sites identified by the Roadmap Epigenomics Mapping Consortium, we observed strong enrichment of SNP heritability for all five tested neuropsychiatric disorders (but not for the two control traits), with Z-score $p$-values surviving Bonferroni correction in each case (Figure 2; Table S2). With the exception of enrichment of SNP heritability for major depressive disorder in fetal brain OCRs overlapping H3K4me3 sites, enrichments within fetal brain OCRs overlapping $\mathrm{H} 3 \mathrm{~K} 4 \mathrm{me} 1$ or $\mathrm{H} 3 \mathrm{~K} 4 \mathrm{me} 3$ regions were consistently higher than within fetal brain OCRs or histone modification sites alone (Figure 3), highlighting the value of additional epigenomic annotations to define regulatory regions of the genome.

In the adult brain, common genetic risk for schizophrenia has been reported to be primarily mediated through OCRs in $\mathrm{NeuN}+$ (neuronal), rather than $\mathrm{NeuN}_{-}$(nonneuronal), nuclei (Fullard et al., 2018). To explore the cellular basis of genetic risk for neuropsychiatric disorders in the prenatal brain, we determined which of our fetal frontal cortex OCRs could be confidently attributed to $\mathrm{NeuN}+$ and $\mathrm{NeuN}$ - fractions by fluorescence-activated sorting nuclei from the same three fetal frontal cortex samples. This identified 30,162 high confidence bulk fetal frontal cortex OCRs that were also observed in sorted $\mathrm{NeuN}+$ (neuron-enriched) nuclei (at FDR $<0.01$ ) and 37,576 such OCRs that were also observed in sorted NeuN(neuron-depleted) nuclei (at FDR < 0.01), of which 27,528 OCRs overlapped both $\mathrm{NeuN}+$ and $\mathrm{NeuN}-$ fractions (Figure 4a). OCRs (FDR < 0.01) specific to NeuN + nuclei included sites at the proximal promoter of GABRB3 ( $-141 \mathrm{bp}$ from the TSS) and in Intron 1 of the neuronal marker MAP2 ( $+1,614 \mathrm{bp}$ from the TSS). OCRs (FDR < 0.01$)$ identified in $\mathrm{NeuN}$ - but not $\mathrm{NeuN}+$ nuclei include sites at the proximal promoter of the gene encoding the oligodendrocyte precursor cell marker OLIG2 (-139 bp from the TSS), and sites at the proximal promoters of the genes encoding the neural progenitor/radial glia cell markers nestin (NES; $-399 \mathrm{bp}$ from the TSS), SOX9 (-46 bp from the TSS), and SOX2 (-63 bp from the TSS; Figure 4b), consistent with successful depletion of nonneuronal nuclei in the $\mathrm{NeuN}+$ fraction.

We observed significant enrichment of SNP heritability for schizophrenia in OCRs identified in fetal frontal cortex NeuN+ nuclei, which survived correction for multiple testing (6.5-fold enrichment, Zscore $\left.p=4.16 \times 10^{-4}\right)$. Moreover, SNP heritability for schizophrenia, bipolar disorder, and major depressive disorder was enriched at Bonferroni-corrected significance in $\mathrm{NeuN}+$ OCRs overlapping either

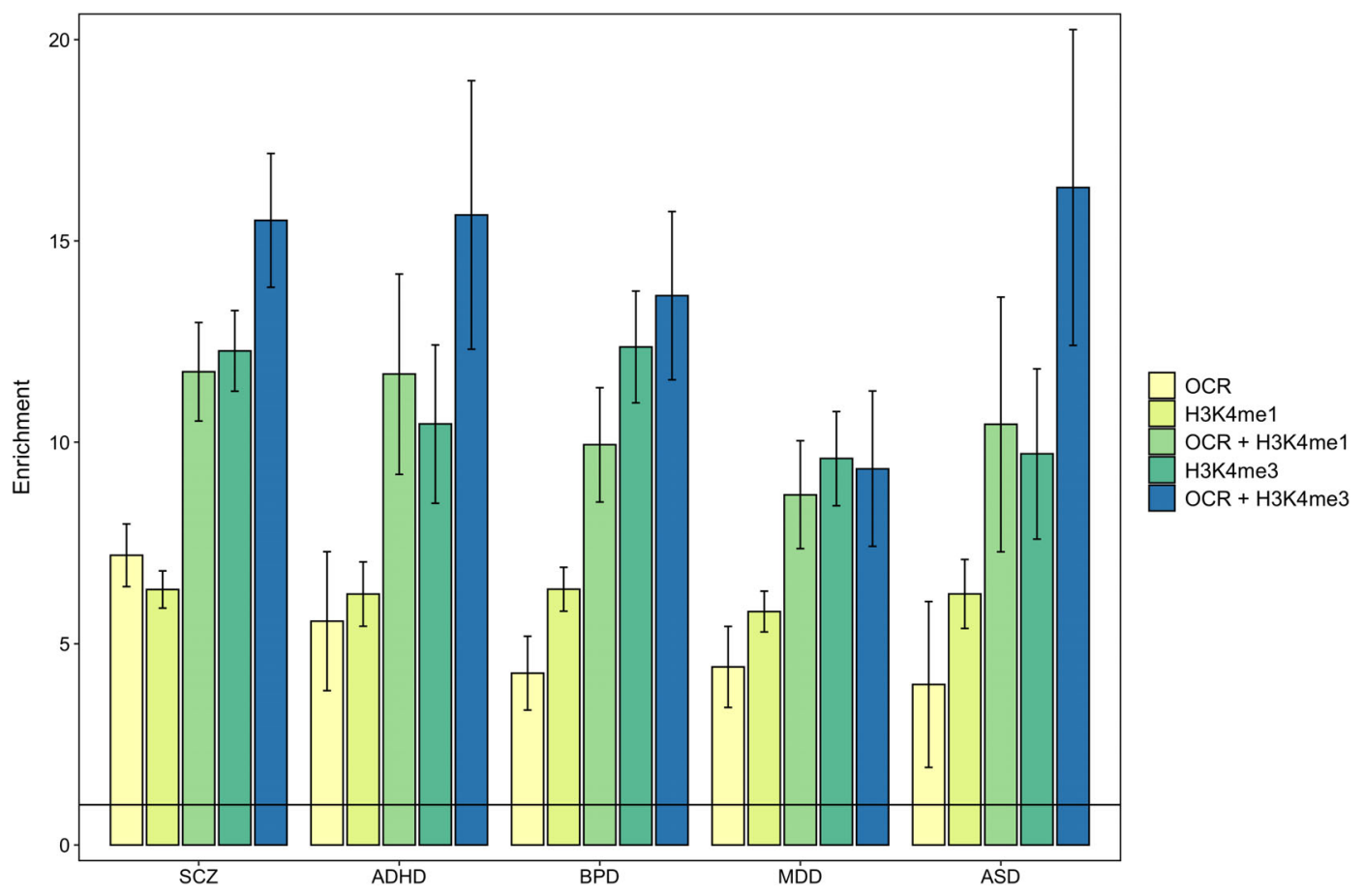

FIGURE 3 Enrichment of SNP heritability for five neuropsychiatric disorders within open chromatin regions identified in bulk fetal frontal cortex (OCR), within previously identified sites of histone modification in the human fetal brain (H3K4me1 and H3K4me3) and within bulk fetal frontal cortex open chromatin regions overlapping each histone modification (OCR + H3K4me1 and OCR + H3K4me3). Fold-enrichment estimate of SNP heritability for each trait is the proportion of SNP heritability explained by SNPs within the annotation divided by the proportion of genome-wide SNPs within the annotation. The solid horizontal line indicates no enrichment. Error bars represent standard error. ADHD, attention deficit hyperactivity disorder; ASD, autism spectrum disorder; BLD TG, blood triglyceride levels; MDD, major depressive disorder; SCZ, schizophrenia 
(a)

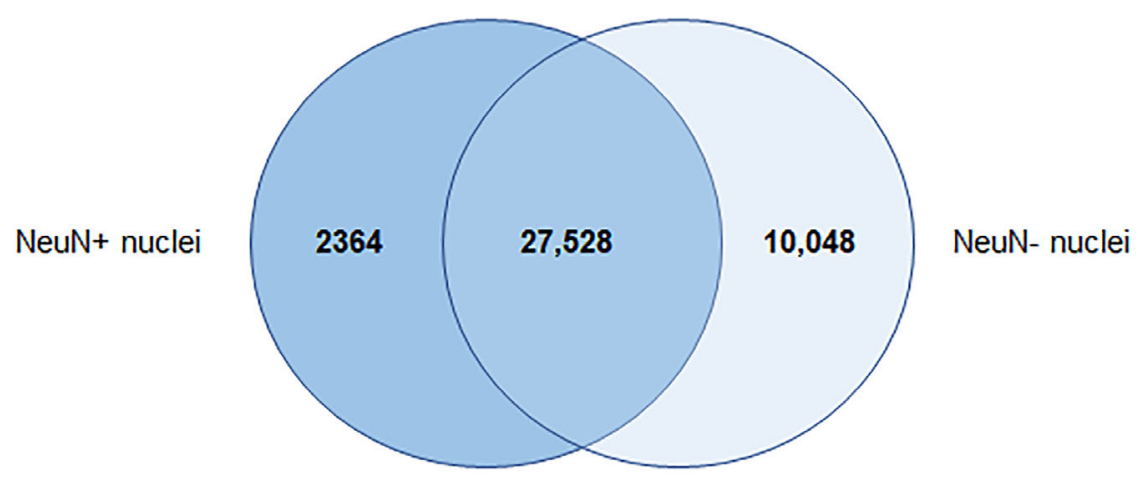

(b)

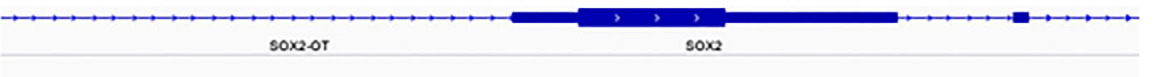

NeuN+ nuclei
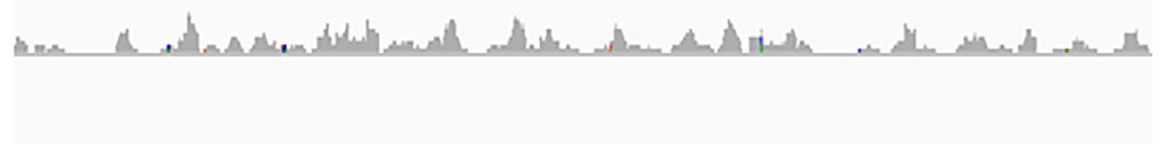

NeuN- nuclei

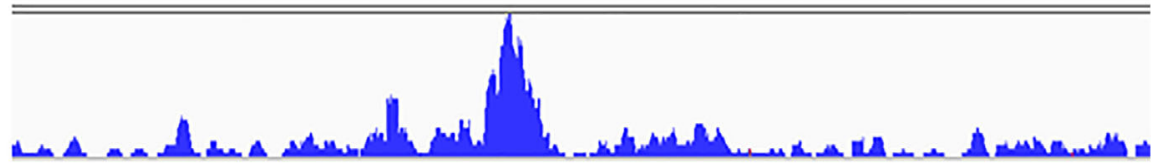

FIGURE 4 High confidence open chromatin regions identified in NeuN+ and NeuN- nuclei from the fetal frontal cortex. (a) Overlap between open chromatin regions identified in fetal frontal cortex $\mathrm{NeuN}+$ and $\mathrm{NeuN}$ - nuclei. (b) Combined reads from the three fetal $\mathrm{NeuN}+$ samples and three fetal $\mathrm{NeuN}$ - samples showing a high confidence open chromatin peak at the transcription start site of the SOX2 gene in $\mathrm{NeuN}-$ but not $\mathrm{NeuN}+$ nuclei

fetal H3K4me1 sites (Figure 5) or H3K4me3 sites, with nominally significant enrichments observed for ASD in NeuN+ OCRs overlapping H3K4me1 sites and for ADHD in $\mathrm{NeuN}+$ OCRs overlapping H3K4me3 sites (Table S3). Within fetal frontal cortex NeuN- nuclei, we observed enrichment of SNP heritability for schizophrenia (8.14-fold enrichment, Z-score $p=1.2 \times 10^{-7}$ ) and ADHD (8.69-fold enrichment, $Z$-score $p=1.54 \times 10^{-4}$ ) at $Z$-score $p$-values surviving Bonferroni correction, and for bipolar disorder at nominal significance (5.82-fold enrichment, Z-score $p=.01$ ). For fetal frontal cortex NeuN- nuclei overlapping either fetal H3K4me1 (Figure 6) or $\mathrm{H} 3 \mathrm{~K} 4 \mathrm{me} 3$ sites, we observed significant enrichment of SNP heritability for all five tested neuropsychiatric disorders, each surviving correction for multiple testing (Table S4).

A limitation of GWAS approaches to complex disorders is that the identification of the causal genetic variants underlying associations is often complicated by linkage disequilibrium (resulting in multiple variants at a locus displaying similar levels of association) and incomplete functional characterization of noncoding regions of the genome. To refine potentially functional genetic variants driving genome-wide significant associations with neuropsychiatric disorders in GWAS, we identified SNPs that were located within a bulk fetal frontal cortex OCR, associated with any of the five tested neuropsychiatric conditions at genome-wide significance $(p<5$ $\left.\times 10^{-8}\right)$ and in strong linkage disequilibrium $\left(r^{2}>0.8\right)$ with the GWAS index SNP at the locus. A total of 68 such OCR SNPs were identified for schizophrenia and 15 for bipolar disorder, of which 12 (on Chromosome 6) were shared between the two conditions. We further characterize these OCR SNPs in terms of whether they are also in detected fetal brain H3K4me1 and/or H3K4me3 sites (Roadmap Epigenomics Mapping Consortium, 2015) can be confidently attributed to $\mathrm{NeuN}+$ and/or NeuN - nuclei and if they have been found to be a high confidence eQTL $\left(p<5 \times 10^{-5}\right)$ for any transcripts in human fetal brain (O'Brien et al., 2018) (Table S5). We found that OCR SNPs on Chromosome 6 were not only in strong LD with schizophrenia and bipolar disorder GWAS index SNPs, but also with the most significant eQTL for three transcripts of the BTN2A1, ZSCAN12P1, and H4C13 genes $\left(r^{2}\right.$ with the top eQTL of between 88 and 1 ) in fetal brain. We also observed schizophrenia-associated OCR SNPs on Chromosome 8 associated with fetal brain eQTL for DDHD2 and FGFR1 transcripts ( $r^{2}$ with top eQTL of .85 and .95 , respectively), and on Chromosome 13 associated with fetal brain eQTL for the long noncoding RNAs LINC01068 and LINC01038 ( $r^{2}$ with top eQTL of .88 and 1, respectively; Figure 7). We did not identify any OCR SNPs in strong LD with the GWAS index SNPs at genome-wide significant loci 
(a)

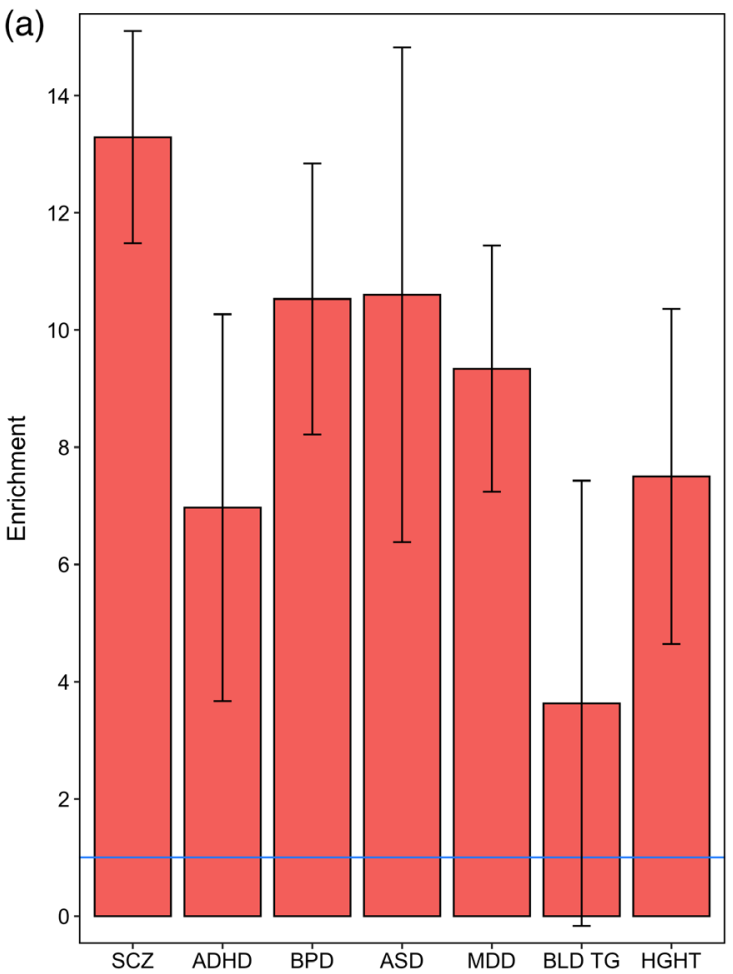

(b)

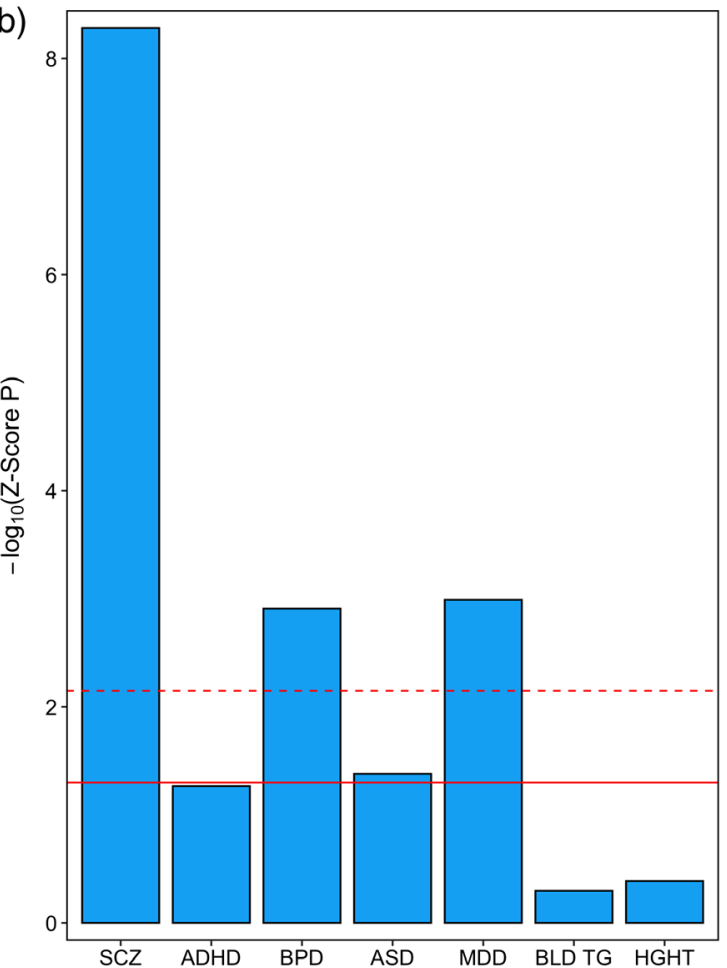

FIGURE 5 Partitioned heritability for five neuropsychiatric disorders and two control traits within high confidence open chromatin regions observed in fetal brain NeuN + nuclei overlapping fetal brain H3K4me1 sites. (a) Fold-enrichment estimates of SNP heritability for each trait (the proportion of SNP heritability explained by SNPs within the annotation divided by the proportion of genome-wide SNPs within the annotation). Error bars represent standard error. The solid horizontal line indicates no enrichment. (b) $-\log _{10} Z$-score $p$-values (two-tailed) for enrichment of SNP heritability, controlling for general genomic annotations included in the baseline model of Finucane et al. (2015). The solid horizontal line indicates the Z-score $p$-value .05 threshold; the dashed horizontal line indicates the threshold for Z-score $p$-values surviving Bonferroni correction for seven tested traits. ADHD, attention deficit hyperactivity disorder; ASD, autism spectrum disorder; BLD TG, blood triglyceride levels; BPD, bipolar disorder; HGHT, height; MDD, major depressive disorder; SCZ, schizophrenia

for ADHD, ASD, or major depressive disorder, although we note that the number of such loci in these GWAS is substantially lower than genome-wide significant loci in the utilized bipolar disorder and schizophrenia GWAS.

\section{DISCUSSION}

In the absence of clear pathology, but a significant genetic component, insights into the underlying biology of neuropsychiatric disorders are likely to be provided by functional maps of the genome in a variety of human neural cell populations. In the present study, we have mapped regions of open chromatin in bulk tissue, NeuN + and $\mathrm{NeuN}$ - nuclei from the prenatal human frontal cortex. By integrating these maps with other epigenomic data from the human fetal brain (Roadmap Epigenomics Consortium, 2015) and summary statistics from recent large-scale GWAS (Demontis et al., 2019; Grove et al., 2019; Mullins et al., 2021; Ripke et al., 2020; Wray et al., 2018), we provide evidence for an early neurodevelopmental component to a range of neuropsychiatric conditions and highlight an important role for regulatory regions active within both $\mathrm{NeuN}+$ and $\mathrm{NeuN}-$ cells of the prenatal brain in susceptibility to these disorders.
Our findings add to growing evidence that a proportion of the common genetic variants conferring risk to neuropsychiatric disorders are active during prenatal brain development (e.g., Clifton et al., 2019; Cross-Disorder Group of the Psychiatric Genomics Consortium, 2019; de la Torre-Ubieta et al., 2018; Forsyth et al., 2020; Hall et al., 2021; Hannon et al., 2016; Hill \& Bray, 2012; Li et al., 2018; O'Brien et al., 2018; Schork et al., 2019). A previous study mapped OCRs in the germinal zone (comprising the ventricular zone, subventricular zone, and intermediate zone) and cortical plate (encompassing the subplate, cortical plate, and marginal zone) of the human fetal cerebral cortex at 15-17 PCW, reporting significant enrichment of SNP heritability for ADHD, depressive symptoms, neuroticism, and schizophrenia within sites that were found to be preferentially accessible in the germinal zone (de la Torre-Ubieta et al., 2018). In the present study, we confirm enrichment of SNP heritability for ADHD, major depressive disorder, and schizophrenia within OCRs of the human (frontal) cortex during the second trimester of gestation and extend this observation to also include bipolar disorder and ASD when either $\mathrm{H} 3 \mathrm{~K} 4 \mathrm{me} 1$ or H3K4me3 sites were additionally considered. Although bipolar disorder is not generally considered to be neurodevelopmental in origin, the present data, showing SNP heritability of bipolar disorder to be enriched within fetal brain OCRs at a similar level to that of 

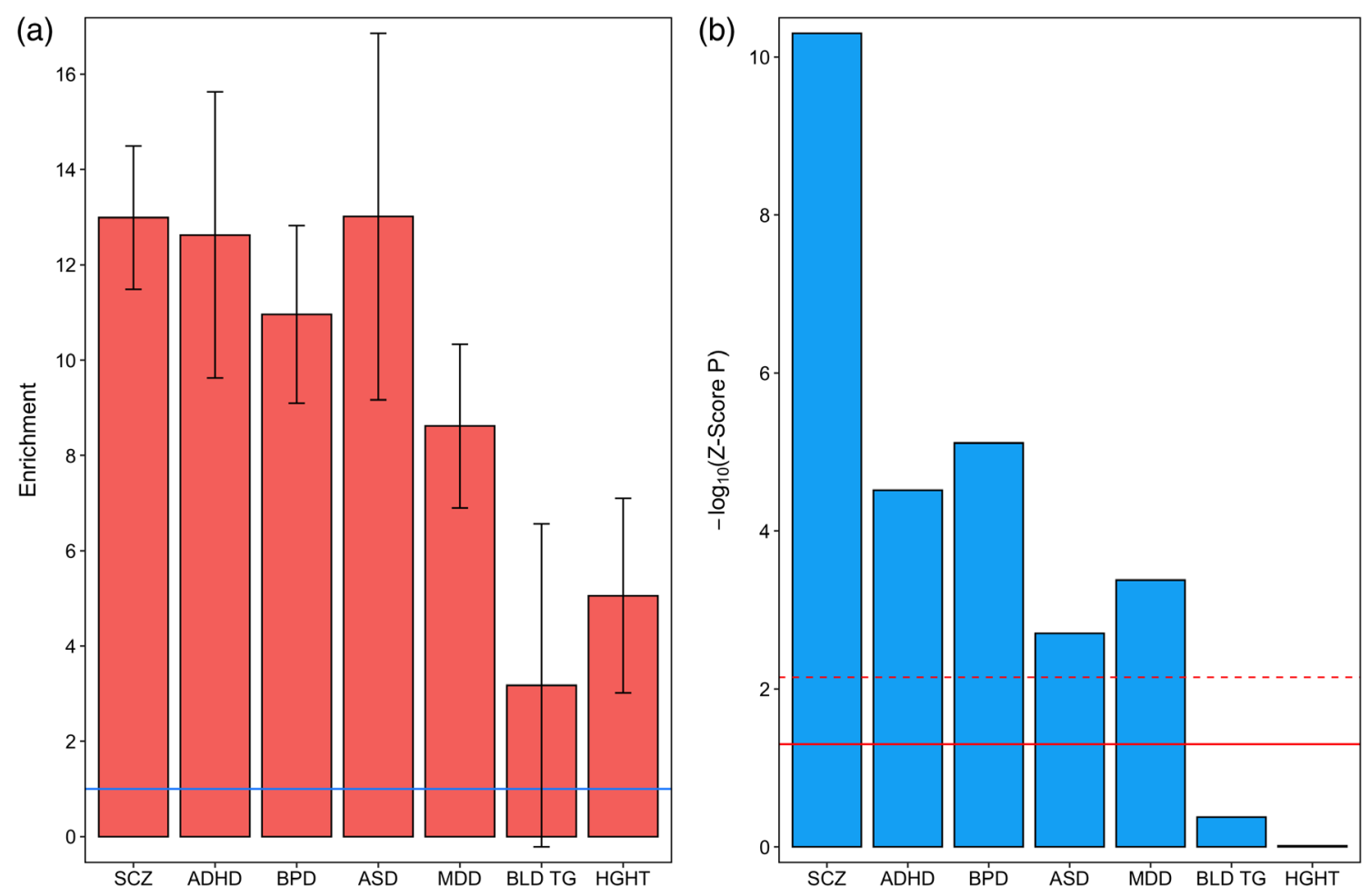

FIG URE 6 Partitioned heritability for five neuropsychiatric disorders and two control traits within high confidence open chromatin regions observed in fetal brain NeuN - nuclei overlapping fetal brain H3K4me1 sites. (a) Fold-enrichment estimates of SNP heritability for each trait (the proportion of SNP heritability explained by SNPs within the annotation divided by the proportion of genome-wide SNPs within the annotation). Error bars represent standard error. The solid horizontal line indicates no enrichment. (b) - $\log _{10} Z$-score $p$-values (two-tailed) for enrichment of SNP heritability, controlling for general genomic annotations included in the baseline model of Finucane et al. (2015). The solid horizontal line indicates the Z-score $p$-value .05 threshold; the dashed horizontal line indicates the threshold for Z-score $p$-values surviving Bonferroni correction for seven tested traits. ADHD, attention deficit hyperactivity disorder; ASD, autism spectrum disorder; BLD TG, blood triglyceride levels; BPD, bipolar disorder; HGHT, height; MDD, major depressive disorder; SCZ, schizophrenia

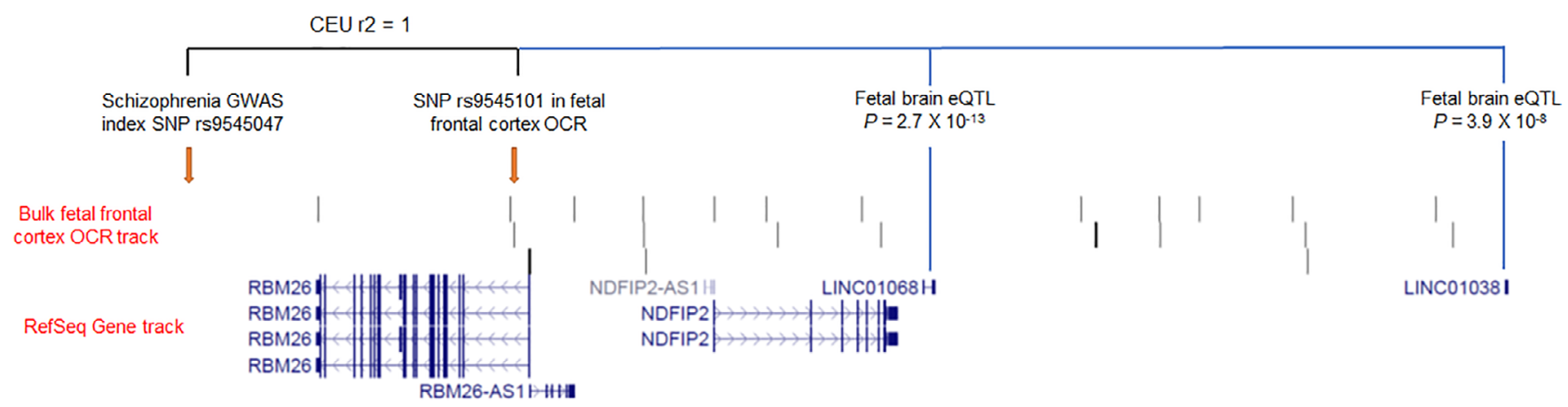

FIGURE 7 Use of open chromatin region maps to identify potentially functional genetic variants at genome-wide association study (GWAS) loci. In this example, a single-nucleotide polymorphism (SNP) within a fetal frontal cortex open chromatin region is in perfect linkage disequilibrium $\left(r^{2}=1\right)$ with the schizophrenia GWAS index SNP at the locus (rs9545047) and is also a significant expression quantitative trait loci for LINC01068 and LINC01038 in the human fetal brain (O'Brien et al., 2018). Image generated using the UCSC Genome Browser (https:// genome.ucsc.edu/index.html) and an uploaded BED file for identified bulk fetal frontal cortex open chromatin regions (available through https:// doi.org/10.6084/m9.figshare.12302387.v1) as a custom track

schizophrenia and ADHD, are consistent with our previous finding of an enrichment of fetal brain eQTL within common genetic risk variants for the condition (O'Brien et al., 2018) and with recent evidence for altered expression of neurodevelopmental genes in iPSC-derived cerebral organoids generated from patients with bipolar disorder
(Kathuria et al., 2020). Emerging evidence suggests that some of the common genetic influences on risk for major depressive disorder also operate in utero (Hall et al., 2021; Wray et al., 2018), although we note that, in the present study, the enrichment of SNP heritability for the condition in fetal brain OCRs was consistently lower than that for 
bipolar disorder and schizophrenia. While rare genetic risk variants for ASD are known to disrupt genes functioning in the prenatal brain (Parikshak et al., 2013; Satterstrom et al., 2020; Willsey et al., 2013), only recently have ASD GWAS yielded sufficient signal for biological insights into the condition (Forsyth et al., 2020; Grove et al., 2019; Hall et al., 2021; Pain et al., 2019).

Our study provides the first maps of open chromatin in $\mathrm{NeuN}+$ and $\mathrm{NeuN}$ - cell populations of the human fetal brain. In contrast to the adult brain, where genetic risk for schizophrenia appears to be largely mediated by NeuN+ cells (Fullard et al., 2018), we find that, within the fetal brain, genetic risk for this and other neuropsychiatric conditions is also strongly enriched within high confidence OCRs observed in NeuN- nuclei. However, whereas NeuN- cells in the adult brain largely consist of mature glia (astrocytes and oligodendrocytes), those of the second-trimester fetal brain encompass a variety of developing cells, including intermediate progenitors, radial glia, and oligodendrocyte precursors. Our data are therefore consistent with the findings of de la Torre-Ubieta et al. (2018), who report enrichment of SNP heritability for neuropsychiatric disorders in OCRs of the neural progenitor cell-containing germinal zone, and Schork et al. (2019), who report enriched expression of fine-mapped candidates genes for a broad neuropsychiatric phenotype in fetal radial glia. However, it is likely that the NeuN - nuclei assayed in this study also include nuclei from neurons at early stages of development. Mapping of OCRs in human prenatal brain using single-cell/nuclei sequencing technologies will be necessary to elucidate the specific fetal cell populations relevant to neuropsychiatric disorders.

We used our high confidence fetal frontal cortex OCRs to identify potentially functional noncoding SNPs tagged by genome-wide significant risk SNPs for schizophrenia and bipolar disorder, which may serve as a guide for future functional studies (e.g., genome editing). Although we have focused on neuropsychiatric phenotypes, our maps of open chromatin in the fetal frontal cortex are likely to be useful in exploring early neurodevelopmental antecedents to a variety of brainrelated conditions. As we move into the era of whole genome sequencing, maps of functional elements within the noncoding genome will continue to be important. We therefore provide the genomic coordinates of our high confidence fetal brain OCRs through a publicly accessible online repository (https://doi.org/10.6084/m9. figshare.12302387.v1) for use by the research community.

\section{ACKNOWLEDGMENTS}

This work was supported by a Medical Research Council (MRC) GW4 BioMed Doctoral Training Partnership studentship to M.R.K., an MRC project grant to N.J.B. (grant no. MR/L010674/2), and an MRC Centre (grant no. MR/L010305/1). The human fetal material was provided by the Joint MRC/Wellcome Trust (grant \#099175/Z/12/Z) Human Developmental Biology Resource (https://www.hdbr.org). The authors would like to thank Dr Joe Burrage, Dr Catherine Naseriyan, and Dr Ann KiftMorgan for assistance with fluorescence-activated nuclei sorting, and Dr Joanne Morgan for assistance with sequencing. They would also like to thank the schizophrenia working group of the Psychiatric Genomics Consortium for use of summary GWAS data prior to publication, and the research participants and employees of 23andMe for their contribution to the major depressive disorder GWAS data used in this study. Comparison adult frontal cortex ATAC-Seq data were generated as part of the CommonMind Consortium, supported by funding from Takeda Pharmaceuticals Company Limited, F. Hoffmann-La Roche Ltd, and NIH grants R01MH085542, R01MH093725, P5OMH066392, P5OMH080405, R01MH097276, RO1-MH-075916, P50M096891, P50MH084053S1, R37MH057881, AG02219, AG05138, MH06692, R01MH110921, R01MH109677, R01MH109897, U01MH103392, and contract HHSN271201300031C through IRP NIMH. CMC Leadership: Panos Roussos, Joseph Buxbaum, Andrew Chess, Schahram Akbarian, Vahram Haroutunian (Icahn School of Medicine at Mount Sinai), Bernie Devlin, David Lewis (University of Pittsburgh), Raquel Gur, Chang-Gyu Hahn (University of Pennsylvania), Enrico Domenici (University of Trento), Mette A. Peters, Solveig Sieberts (Sage Bionetworks), Thomas Lehner, Stefano Marenco, Barbara K. Lipska (NIMH).

\section{CONFLICT OF INTEREST}

The authors declare no conflict of interest in relation to this work.

\section{AUTHOR CONTRIBUTIONS}

Laboratory work was performed by Manuela R. Kouakou, under the supervision of Nicholas J. Bray, Matthew J. Hill, Jonathan Mill, and Emma L. Dempster. Primary analyses were performed by Manuela R. Kouakou, with additional analyses performed by Darren Cameron, Eilis Hannon, and Nicholas J. Bray. The manuscript was written by Manuela R. Kouakou and Nicholas J. Bray, with input from all other authors. All authors approved the final version of the manuscript.

\section{DATA AVAILABILITY STATEMENT}

The genomic coordinates of the fetal frontal cortex open chromatin regions defined and used in this study are provided as BED files in a publicly-accessible online repository: https://doi.org/10.6084/m9. figshare.12302387.v1.

\section{ORCID}

Nicholas J. Bray (D) https://orcid.org/0000-0002-4357-574X

\section{REFERENCES}

Bryois, J., Garrett, M. E., Song, L., Safi, A., Giusti-Rodriguez, P., Johnson, G. D., ... Crawford, G. E. (2018). Evaluation of chromatin accessibility in prefrontal cortex of individuals with schizophrenia. Nature Communications, 9, 3121. https://doi.org/10.1038/s41467018-05379-y

Buenrostro, J. D., Giresi, P. G., Zaba, L. C., Chang, H. Y., \& Greenleaf, W. J. (2013). Transposition of native chromatin for fast and sensitive epigenomic profiling of open chromatin, DNA-binding proteins and nucleosome position. Nature Methods, 10, 1213-1218. https://doi.org/10. 1038/nmeth.2688

Clifton, N. E., Hannon, E., Harwood, J. C., Di Florio, A., Thomas, K. L., Holmans, P. A., ... Hall, J. (2019). Dynamic expression of genes associated with schizophrenia and bipolar disorder across development. Translational Psychiatry, 9, 74. https://doi.org/10.1038/s41398-0190405-x 
Courchesne, E., Gazestani, V. H., \& Lewis, N. E. (2020). Prenatal origins of ASD: The when, what, and how of ASD development. Trends in Neurosciences, 43, 326-342. https://doi.org/10.1016/j.tins.2020. 03.005

Cross-Disorder Group of the Psychiatric Genomics Consortium. (2019). Genomic relationships, novel loci, and pleiotropic mechanisms across eight psychiatric disorders. Cell, 179, 1469-1482. https://doi.org/10. 1016/j.cell.2019.11.020

de la Torre-Ubieta, L., Stein, J. L., Won, H., Opland, C. K., Liang, D., Lu, D., \& Geschwind, D. H. (2018). The dynamic landscape of open chromatin during human cortical neurogenesis. Cell, 172, 289-304. https://doi.org/10.1016/j.cell.2017.12.014

Demontis, D., Walters, R. K., Martin, J., Mattheisen, M., Als, T. D., Agerbo, E., ... Neale, B. M. (2019). Discovery of the first genome-wide significant risk loci for attention deficit/hyperactivity disorder. Nature Genetics, 51, 63-75. https://doi.org/10.1038/s41588-018-0269-7

ENCODE Project Consortium. (2012). An integrated encyclopedia of DNA elements in the human genome. Nature, 489, 57-74.

Finucane, H. K., Bulik-Sullivan, B., Gusev, A., Trynka, G., Reshef, Y., Loh, P. R., ... Price, A. L. (2015). Partitioning heritability by functional annotation using genome-wide association summary statistics. Nature Genetics, 47, 1228-1235. https://doi.org/10.1038/ng.3404

Forsyth, J. K., Nachun, D., Gandal, M. J., Geschwind, D. H., Anderson, A. E., Coppola, G., \& Bearden, C. E. (2020). Synaptic and gene regulatory mechanisms in schizophrenia, autism, and 22q11.2 copy number variant-mediated risk for neuropsychiatric disorders. Biological Psychiatry, 87, 150-163. https://doi.org/10.1016/j.biopsych.2019.06.029

Fullard, J. F., Hauberg, M. E., Bendl, J., Egervari, G., Cirnaru, M. D., Reach, S. M., ... Roussos, P. (2018). An atlas of chromatin accessibility in the adult human brain. Genome Research, 28, 1243-1252. https:// doi.org/10.1101/gr.232488.117

Grove, J., Ripke, S., Als, T. D., Mattheisen, M., Walters, R. K., Won, H., ... Børglum, A. D. (2019). Identification of common genetic risk variants for autism spectrum disorder. Nature Genetics, 51, 431-444. https:// doi.org/10.1038/s41588-019-0344-8

Hall, L. S., Pain, O., O'Brien, H. E., Anney, R., Walters, J. T. R., Owen, M. J., ... Bray, N. J. (2021). Cis-effects on gene expression in the human prenatal brain associated with genetic risk for neuropsychiatric disorders. Molecular Psychiatry, 26, 2082-2088. https://doi.org/10.1038/ s41380-020-0743-3

Hannon, E., Spiers, H., Viana, J., Pidsley, R., Burrage, J., Murphy, T. M., ... Mill, J. (2016). Methylation QTLs in the developing brain and their enrichment in schizophrenia risk loci. Nature Neuroscience, 19, 48-54. https://doi.org/10.1038/nn.4182

Hill, M. J., \& Bray, N. J. (2012). Evidence that schizophrenia risk variation in the ZNF804A gene exerts its effects during fetal brain development. American Journal of Psychiatry, 169, 1301-1308. https://doi.org/10. 1176/appi.ajp.2012.11121845

Hoffman, G. E., Bendl, J., Voloudakis, G., Montgomery, K. S., Sloofman, L., Wang, Y. C., ... Roussos, P. (2019). CommonMind consortium provides transcriptomic and epigenomic data for schizophrenia and bipolar disorder. Scientific Data, 6, 180. https://doi.org/10.1038/s41597-0190183-6

lotchkova, V., Ritchie, G. R. S., Geihs, M., Morganella, S., Min, J. L., Walter, K., ... Soranzo, N. (2019). GARFIELD classifies disease-relevant genomic features through integration of functional annotations with association signals. Nature Genetics, 51, 343-353. https://doi.org/10. 1038/s41588-018-0322-6

Kathuria, A., Lopez-Lengowski, K., Vater, M., McPhie, D., Cohen, B. M., \& Karmacharya, R. (2020). Transcriptome analysis and functional characterization of cerebral organoids in bipolar disorder. Genome Medicine, 12, 34. https://doi.org/10.1186/s13073-020-00733-6

Langmead, B., Trapnell, C., Pop, M., \& Salzberg, S. L. (2009). Ultrafast and memory-efficient alignment of short DNA sequences to the human genome. Genome Biology, 10, R25. https://doi.org/10.1186/gb-200910-3-r25

Lango Allen, H., Estrada, K., Lettre, G., Berndt, S. I., Weedon, M. N., Rivadeneira, F., ... Hirschhorn, J. N. (2010). Hundreds of variants clustered in genomic loci and biological pathways affect human height. Nature, 467, 832-838. https://doi.org/10.1038/nature09410

Li, H., Handsaker, B., Wysoker, A., Fennell, T., Ruan, J., Homer, N., ... Durbin, R. (2009). The sequence alignment/map format and SAMtools. Bioinformatics, 25, 2078-2079. https://doi.org/10.1093/bioinformatics/btp352

Li, M., Santpere, G., Imamura Kawasawa, Y., Evgrafov, O. V., Gulden, F. O., Pochareddy, S., ... Sestan, N. (2018). Integrative functional genomic analysis of human brain development and neuropsychiatric risks. Science, 362, eaat7615. https://doi.org/10.1126/science.aat7615

Libbrecht, M. W., Rodriguez, O. L., Weng, Z., Bilmes, J. A., Hoffman, M. M., \& Noble, W. S. (2019). A unified encyclopedia of human functional DNA elements through fully automated annotation of 164 human cell types. Genome Biology, 20, 180. https://doi.org/10. 1186/s13059-019-1784-2

Mullins, N., Forstner, A. J., O'Connell, K. S., Coombes, B., Coleman, J., Qiao, Z., ... Andreassen, O. A. (2021). Genome-wide association study of more than 40,000 bipolar disorder cases provides new insights into the underlying biology. Nature Genetics, 53, 817-829. https://doi.org/ 10.1038/s41588-021-00857-4

O'Brien, H. E., Hannon, E., Hill, M. J., Toste, C. C., Robertson, M. J., Morgan, J. E., ... Bray, N. J. (2018). Expression quantitative trait loci in the developing human brain and their enrichment in neuropsychiatric disorders. Genome Biology, 19, 194. https://doi.org/10.1186/s13059018-1567-1

O'Donnell, K. J., \& Meaney, M. J. (2017). Fetal origins of mental health: The developmental origins of health and disease hypothesis. American Journal of Psychiatry, 174, 319-328. https://doi.org/10.1176/appi.ajp. 2016.16020138

Pain, O., Pocklington, A. J., Holmans, P. A., Bray, N. J., O'Brien, H. E., Hall, L. S., ... Anney, R. (2019). Novel insight into the etiology of autism spectrum disorder gained by integrating expression data with genomewide association statistics. Biological Psychiatry, 86, 265-273. https:// doi.org/10.1016/j.biopsych.2019.04.034

Parikshak, N. N., Luo, R., Zhang, A., Won, H., Lowe, J. K., Chandran, V., ... Geschwind, D. H. (2013). Integrative functional genomic analyses implicate specific molecular pathways, and circuits in autism. Cell, 155, 1008-1021. https://doi.org/10.1016/j.cell.2013.10.031

Quinlan, A. R., \& Hall, I. M. (2010). BEDTools: A flexible suite of utilities for comparing genomic features. Bioinformatics, 26, 841-842. https:// doi.org/10.1093/bioinformatics/btq033

Raudvere, U., Kolberg, L., Kuzmin, I., Arak, T., Adler, P., Peterson, H., \& Vilo, J. (2019). g:Profiler: A web server for functional enrichment analysis and conversions of gene lists (2019 update). Nucleic Acids Research, 47, W191-W198. https://doi.org/10.1093/nar/gkz369

Ripke, S., Walters, J. T. R., \& O'Donovan, M. C. (2020). Mapping genomic loci prioritises genes and implicates synaptic biology in schizophrenia. medRXiv preprint. https://doi.org/10.1101/2020.09.12.20192922

Roadmap Epigenomics Consortium. (2015). Integrative analysis of 111 reference human epigenomes. Nature, 518, 317-330. https://doi.org/10. 1038/nature14248

Satterstrom, F. K., Kosmicki, J. A., Wang, J., Breen, M. S., De Rubeis, S., An, J. Y., ... Buxbaum, J. D. (2020). Large-scale exome sequencing study implicates both developmental and functional changes in the neurobiology of autism. Cell, 180, 568-584. https://doi.org/10.1016/ j.cell.2019.12.036

Schork, A. J., Won, H., Appadurai, V., Nudel, R., Gandal, M., Delaneau, O., ... Werge, T. (2019). A genome-wide association study of shared risk across psychiatric disorders implicates gene regulation during fetal neurodevelopment. Nature Neuroscience, 22, 353-361. https://doi. org/10.1038/s41593-018-0320-0 
Song, L., Zhang, Z., Grasfeder, L. L., Boyle, A. P., Giresi, P. G., Lee, B. K., ... Furey, T. S. (2011). Open chromatin defined by DNasel and FAIRE identifies regulatory elements that shape cell-type identity. Genome Research, 21, 1757-1767. https://doi.org/10.1101/gr.121541.111

Stark, R., \& Brown, G. (2011). DiffBind: Differential binding analysis of ChIP-Seq peak data. http://bioconductor.org/packages/release/bioc/ vignettes/DiffBind/inst/doc/DiffBind.pdf

Teslovich, T. M., Musunuru, K., Smith, A. V., Edmondson, A. C., Stylianou, I. M., Koseki, M., ... Kathiresan, S. (2010). Biological, clinical and population relevance of 95 loci for blood lipids. Nature, 466, 707713. https://doi.org/10.1038/nature09270

Tsompana, M., \& Buck, M. J. (2014). Chromatin accessibility: A window into the genome. Epigenetics and Chromatin, 7, 33. https://doi.org/10. 1186/1756-8935-7-33

Weinberger, D. R. (1987). Implications of normal brain development for the pathogenesis of schizophrenia. Archives of General Psychiatry, 44, 660-669. https://doi.org/10.1001/archpsyc.1987.01800190080012

Willsey, A. J., Sanders, S. J., Li, M., Dong, S., Tebbenkamp, A. T., Muhle, R. A., ... State, M. W. (2013). Coexpression networks implicate human midfetal deep cortical projection neurons in the pathogenesis of autism. Cell, 155, 997-1007. https://doi.org/10.1016/j.cell.2013. 10.020

Wray, N. R., Ripke, S., Mattheisen, M., Trzaskowski, M., Byrne, E. M., Abdellaoui, A., ... Major Depressive Disorder Working Group of the Psychiatric Genomics Consortium. (2018). Genome-wide association analyses identify 44 risk variants and refine the genetic architecture of major depression. Nature Genetics, 50, 668-681. https://doi.org/10. 1038/s41588-018-0090-3
Yu, G., Wang, L. G., \& He, Q. Y. (2015). ChIPseeker: An R/bioconductor package for ChIP peak annotation, comparison and visualization. Bioinformatics, 31, 2382-2383. https://doi.org/10.1093/bioinformatics/ btv145

Zhang, Y., Liu, T., Meyer, C. A., Eeckhoute, J., Johnson, D. S., Bernstein, B. E., ... Liu, X. S. (2008). Model-based analysis of ChIP-Seq (MACS). Genome Biology, 9, R137. https://doi.org/10.1186/gb-20089-9-r137

Zhu, L. J., Gazin, C., Lawson, N. D., Pagès, H., Lin, S. M., Lapointe, D. S., \& Green, M. R. (2010). ChIPpeakAnno: A bioconductor package to annotate ChIP-seq and ChIP-chip data. BMC Bioinformatics, 11, 237. https://doi.org/10.1186/1471-2105-11-237

\section{SUPPORTING INFORMATION}

Additional supporting information may be found in the online version of the article at the publisher's website.

How to cite this article: Kouakou, M. R., Cameron, D., Hannon, E., Dempster, E. L., Mill, J., Hill, M. J., \& Bray, N. J. (2021). Sites of active gene regulation in the prenatal frontal cortex and their role in neuropsychiatric disorders. American Journal of Medical Genetics Part B: Neuropsychiatric Genetics, 186B:376-388. https://doi.org/10.1002/ajmg.b.32877 